\title{
Novel NoMix toilet concept for efficient separation of urine and feces and its design optimization using computational fluid mechanics
}

\author{
Joel Gundlach ${ }^{1,}{ }^{*}$, Michael Bryla², Tove A. Larsen³, Lotte Kristoferitsch², Harald \\ Gründl ${ }^{2}$, and Markus Holzner ${ }^{1}$ \\ ${ }^{1}$ Institute of Environmental Engineering, ETH Zurich, Wolfgang-Pauli-Str. 15, 8093 Zurich, Switzerland \\ ${ }^{2}$ EOOS Design GmbH, Zelinkagasse 2/6, 1010 Vienna, Austria \\ ${ }^{3}$ Eawag: Swiss Federal Institute of Aquatic Science and Technology, Ueberlandstrasse 133, 8600 Duebendorf, Switzerland \\ *Corresponding author: Joel Gundlach. E-mail: gujoel@ethz.ch, HIL G34.2, Stefano-Franscini-Platz 5, 8093 \\ Zürich, Switzerland
}

\begin{abstract}
Separation of urine from feces in NoMix toilets bears a large potential for mitigating problems associated with the efficient reuse of excreta. A challenge lies in the design of these toilets that should maximize the amount of collected urine while at the same time minimizing its mixing with flush water that partly spills into the urine collector. We present a novel design idea that takes advantage of the so-called tea pot effect, which describes the attachment of liquids to a curved wall due to surface tension. This effect is used to efficiently guide the urine along a curved entrance into the collector. Computational Fluid Dynamics (CFD) simulations of the two phase (water/urine - air) system are carried out on different toilet geometries where we vary the inflow to the urine collector. In particular, we investigate two different radial curvatures and two V-profile grooves whose combinations result in four toilet geometries that are tested. We demonstrate that the urine collection can be dramatically improved by tailoring curvature radius and V-profile grooves, while keeping the amount of flush water in the urine collector at a minimum. A reduction to less the $2.5 \%$ of flush water entering the urine collector could be achieved by implementing a curvature radius of $15 \mathrm{~mm}$ and a V-profile groove width of $60 \mathrm{~mm}$. The improved geometry's performance makes it an appealing candidate for broad application both in western and developing counties and we anticipate that this technology might replace traditional toilet designs in the future.
\end{abstract}

Keywords - Multiphase Flow, Numerical Simulation, Sustainable building technology

\section{INTRODUCTION}

Closing cycles of resources is getting more and more into the focus of scientific research and industrial applications. Similarly, the perception of waste is changing and what used to be declared non-reusable often becomes a valuable resource. Both aspects are highly relevant to the sanitation sector, where researchers aim at creating an efficient reuse of human excreta. Separating urine, feces, and flush water could minimize entropy in the system and open the doors for new technologies allowing a sustainable and economically interesting use of human excreta [1,2]. One of the major challenges following this idea is the separation of urine from flush water at the source, i.e. the NoMix concept. Urine is a concentrated nutrient solution containing about $90 \%$ of the nitrogen and $60 \%$ of the phosphorus excreted by humans [3]. Without urine, the biological wastewater treatment process would be more or less nutrient-balanced or even nutrient-limited $[3,4]$. This means that with

This document is the accepted manuscript version of the following article:

Gundlach, J., Bryla, M., Larsen, T. A., Kristoferitsch, L., Gründ1, H., \& Holzner, M. (2020). Novel NoMix toilet concept for efficient separation of urine and feces and its design optimization using computational fluid mechanics. Journal of Building Engineering, 101500. https://doi.org/10.1016/j.jobe.2020.101500

This manuscript version is made available under the CC-BY-NC-ND 4.0

license http://creativecommons.org/1icenses/by-nc-nd/4.0/ 
urine separation, simple treatment plants can produce an effluent comparable to the most advanced nutrient eliminating treatment plants existing [4]. Many authors have discussed the merits of urine separation for recycling nutrients to agriculture, e.g. $[1,2,5]$, and the technical possibilities for urine treatment $[6,7]$. Furthermore, urine contains about two thirds of the pharmaceuticals and hormones ending up in wastewater [8]. Increasing awareness of the negative effects of these compounds on aquatic ecosystems will move rational decisions towards urine separation [9]. Lienert and Larsen $[10,11]$ have shown that the acceptance of urine separation is generally very high, but that practical problems with NoMix toilets drastically decrease the readiness of consumers to live with this technology in their own homes. In Switzerland, we found that the most important argument for being in favor of urine separation is nutrient recycling [10], and a meta-analysis revealed that in Europe about 85\% of the surveyed population found a urine-based fertilizer a good idea [11]. However, in some countries farmers will only accept a urine-based fertilizer if it is sanitized and free of pharmaceuticals and hormones [12]. By now, Swiss authorities have approved a urine-based fertilizer fulfilling these requirements for use on all crops [13]. In different settings with NoMix toilets, Rossi et al. [14] estimated that with existing urine separating toilets, the recovery of urine was at the most $70-75 \%$, albeit this estimate is highly uncertain. One of the problems observed was the malfunctioning of the valves used for avoiding the mixing of urine and flush water. The main bottleneck for implementation of urine source separation is thus the manufacturing of NoMix toilets, which are at the same time easy to maintain and highly accepted by consumers. The toilet discussed in this paper has the potential to meet these criteria because it demands no behavioral change from the users and functions without valves or any other moving parts. Given that approximately half of all nonrenewable resources (water, energy, and materials) consumed by human activities are used in construction $[15,16]$, our waste- and water-efficient toilet design may thus contribute to a more sustainable and energy efficient building technology.

Next to experimental testing, Computational Fluid Dynamics (CFD) analysis has proven a useful tool for designing toilet geometries. CFD-analysis reduces the time and cost of prototyping and is a proven method to analyse and visualize multiphase flows. It is commonly used to investigate toilet performances and several studies have been conducted where toilet flushing was analysed via CFD-simulations [17-19].

In this paper we describe a new approach to achieve separation of urine and flush water in an inventive way by changing the toilets shape and making use of the so-called teapot effect [20,21]. Hereby, the urine is directed around a mild curvature into a urine collector, whereas the flush water detaches at the curvature and flows into the bottom outlet of the toilet (Fig. 1). Next to the curvature, we also implement V-shaped surface grooves to optimally guide the urine over the curvature. To study the system performance, CFD-analysis were conducted via Ansys fluent $\mathrm{R}$ [22] simulating the multiphase (liquid-air) flow. We investigate two different radial curvatures and two V-profile grooves whose different combination result in four prototypes that are tested. We demonstrate that the urine collection is efficient on all four tested geometries and can be maximized with a particular choice of curvature radius and grooves, while minimizing the mixing with flush water.

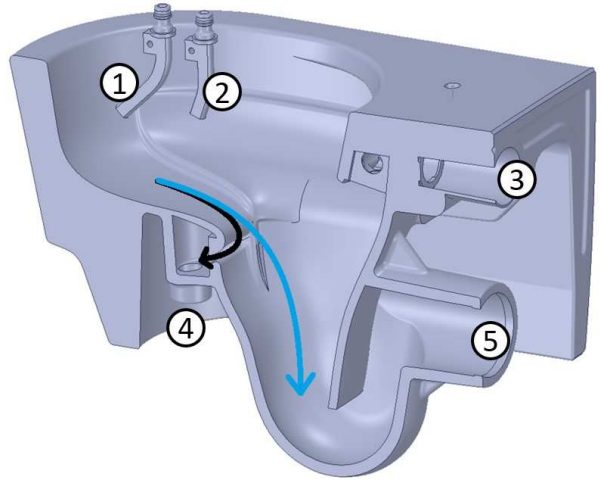


Figure 1: Schematic illustration presenting the general principle of urine separation (demonstrated on Prototype 4). The black arrow shows the hypothetical urine flow direction, the blue one the flush water flow-stream. 1 male urine inlet, 2 female urine inlet, 3 fush inlet, 4 urine outlet, 5 main outlet

\section{Methodology}

\subsection{Prototyping}

The initial set up which constitutes the basis for our investigations consists of a toilet geometry and two tailored male and female urine inlets - termed Uromat (Fig. 1). The position and shape of these inlets are the outcome of a separate study by EOOS in which they investigated the best model inlet based on thermal imaging of several male and female test persons [23].

Our novel idea for an efficient urine separation is that the urine is directed over a curvature, which should have an adequate radius to guide urine into the urine collector, while flush water should detach. In addition, we employ V-profile grooves, channelling the fluid over the curvature (Fig. 2). How strongly a fluid attaches to a curvature -known as teapot effect- depends on several parameters such as the curvatures radius, flow velocity, film thickness, fluid density and surface tension forces [20,21]. Duez et. al. [20] have demonstrated that the critical Weber number, $W e_{c}$, where a liquid detaches, scales as

$$
W e_{c} \propto \frac{r_{i} R}{e_{0}^{2}}(1+\cos \Theta)
$$

with the radius of curvature of the flow streamlines $(R)$, the radius of curvatures of the spout $\left(r_{i}\right)$, the film thickness $\left(e_{0}\right)$ and the contact angle $\Theta_{0}[20]$. The physical interpretation of Eq. (1) is that a fluid is less likely to detach from a curved boundary (i.e. high $W e_{c}$ ), when the curvatures of flow streamlines and spout are large, the fluid film is thin, and the surface tension is high. We are incorporating two different V-profile grooves and two different radial curvatures (Fig. 2) with the aim of controlling the film thickness and the curvature radius to achieve a separation of the more gently flowing urine from the flush water. That is, we aim for close to perfect attachment for the urine so that it flows into the urine collector, while flush water conditions should be above the critical Weber number and separate from the curvature so that it is diverted into the main outlet.
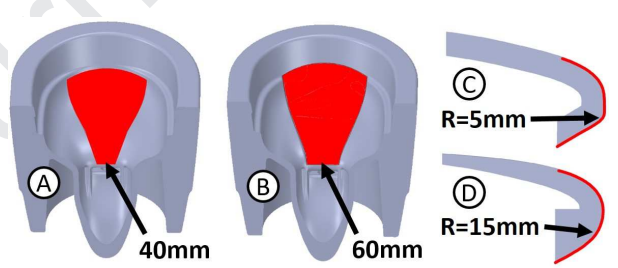

Figure 2: Different V-profiles (width in [mm]) and curvatures (Radius of curvature in [mm]) that were built into the prototypes. Prototype $1(A+C)$, Prototype $2(B+C)$, Prototype $3(A+D)$, Prototype $4(B+D)$

We analyzed the four resulting geometries (Prototype 1-4) according to their performance with the male and female urine flow and the flush. We also constructed a real model of Prototype 3 which was used to validate the model results. In the following, the simulation workflow and experimental conditions will be explained in detail.

\subsection{Simulation workflow}

For each of the four prototypes, we used the following workflow:

1) Create a CAD file containing the geometry (Fig. 3.1) 
2) Create the flow sphere (Fig. 3.2)

3) Meshing of the flow sphere (Fig. 3.3)

4) Run the simulation (Fig. 3.4)

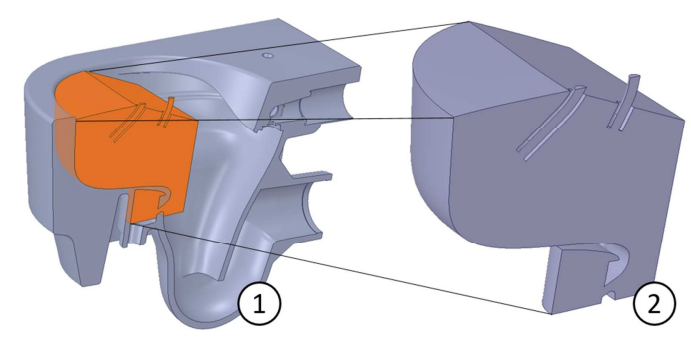

Figure3:IllustrativeexampleoftheworkflowfromtheCADfile (3) tothesimulationresult (4) onPrototype4(urinemale).
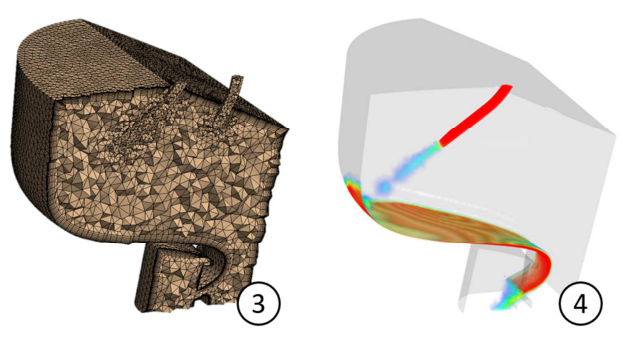

(1) overtheflowsphere

\subsubsection{CAD files}

All CAD files were designed via Solidworks ${ }^{\circledR}[24]$.

\subsubsection{Creation of the simulation sphere}

Since we are interested in the fluid behaviour inside the toilet, only the inner cavity is extracted from the CAD file and used as flow domain (for this step we have used the SpaceClaim ${ }^{\mathrm{R}}$ software [25]). Hereby we only choose the areas of interest and discard all unnecessary regions (Fig. 3) to minimize the computational demand. The obtained volume is then transferred for meshing. As the urine does not occupy the same areas as the flush inside a toilet, the two cases are treated separately to reduce computational cost.

\subsubsection{Meshing}

An adequate mesh is crucial to the quality of the simulation results. It should therefore be handled with care to make sure that it has the right resolution to represent the main physics correctly. Mesh resolution, number of inflation layers, captured curvature and other parameters are optimized to obtain a mesh that is able to capture the flow behaviour correctly, while keeping the number of elements in the mesh reasonably low. All meshes were build via ANSYS Meshing ${ }^{\mathrm{R}}$. The default element size was set to $5 \mathrm{~mm}$, with a maximum size of $6 \mathrm{~mm}$. The minimal defeature size was set to $2 \mathrm{~mm}$ and curvatures were captured down to $2 \mathrm{~mm}$. Although identical parameters were chosen for all meshes, due to the changes in geometry, the number of nodes varied from $2 \cdot 10^{5}$ to $2.4 \cdot 10^{5}$ resulting in a total number of elements between $5.55 \cdot 10^{5}$ and $6.8 \cdot 10^{5}$ depending on the geometry (see supplementary materials for details). We have made convergence studies for selected cases by refining the resolution and verified that the results remained the same.

We have also focused on inflation layers, which are mesh layers created on the boundary to geometry walls (no slip areas) and are used to resolve correctly the velocity transition in this region. We tested 2, 6 and 10 inflation layers and noted that the boundary layer was represented correctly using 10 inflation layers and consequently we used 10 inflation layers for all our simulations.

\subsubsection{Simulation}

The RANS simulations (Reynolds-averaged Navier-Stokes equations) were conducted using Ansys fluent ${ }^{\mathrm{R}}$ [22]. There are numerous models, schemes and parameters that can be selected and adopted. For our simulations we used a transient simulation including a Volume of fluid model for multiphase flow as well as the k-epsilon model 
for turbulence. In addition to these settings, relaxation parameters and time-step/iteration settings were adopted. A full report of the selected parameters can be found in the supplementary materials. All four prototypes were tested under the same conditions, i.e. each case was tested on two different male and female urine flow rates as well as on two different flush rates. Due to the fact, that urine flow rates strongly vary among individuals, we decided to work with the worst case scenario (maximum flow rates implemented as a pulse wave, i.e. we neglect the sharp rising limb and slower recession limb of a real waveform) and assumed a flow rate of $32 \mathrm{ml} / \mathrm{s}$ for a duration of $5 \mathrm{~s}$. The flow rate is on the upper end of peak discharges given in the literature $[26,27]$. The time span of $5 \mathrm{~s}$ is motivated by our observation from initial tests that a steady state is reached fairly quickly and prolonging the duration would not change the main results while costing more computation time. We also run simulations with slightly lower flow rate $(26 \mathrm{ml} / \mathrm{s})$ to prove our hypothesis of better performance at lower flow rates. Due to the fact that we prescribe flow velocities and not flow rates as boundary conditions, the exact flow rate slightly varies between realizations depending on the meshing at the inlet. The flush water was tested on $3.2 \mathrm{~L}$ and $4 \mathrm{~L}$ inflow with an flow rate of $1 \mathrm{~L} / \mathrm{s}$ for a duration of $3.2 \mathrm{~s}$ and $4 \mathrm{~s}$, respectively. The flow rate was estimated reviewing different cistern flush systems. The initial condition in the toilet is set to zero water content and inflow of urine or flush water starts immediately. The simulation time for the flush was set to 8s which was long enough to make sure that all liquid reached the outlets.

Finally, the convergence of residuals is another important aspect of the simulation. In general a fast convergence is desired and can be achieved if the mesh and simulation models/parameters are chosen adequately. However, even with adequate settings, it can take many iterations for convergence to a desired residual value. A time step of $0.1 \mathrm{~ms}$ and 10 iterations per time step provided reasonably fast convergence and high accuracy.

\subsection{Validation experiments}

To validate the conducted CFD-simulations, experiments were carried out on Prototype 3. For these tests, the geometry was build in ceramic and urine flows were introduced via the Uromat [23]. The flow rates were adjusted to match the simulations and water was inserted for about 40 s via the male/female inlets Fig. 4. Subsequently, the fluid entering in the urine collection as well as in the main outlet were quantified by weighting the samples. The long inflow duration was chosen to create bigger samples which minimizes the introduced weighting error calculating the urine fraction. Each experiment was conducted in triplets and averaged (standard variation less then $2 \%$ ). However, large uncertainties exist due to variations in flow rate (manually adjusted), positioning of the Uromat, the sample weighting error, possible impurities of the surface and the fact that in real-test urine is not strictly introduced as plug flow (as in the simulation). These errors are very difficult to quantify which is why we made an educated guess and assumed an total error of roughly $\pm 10 \%$. 


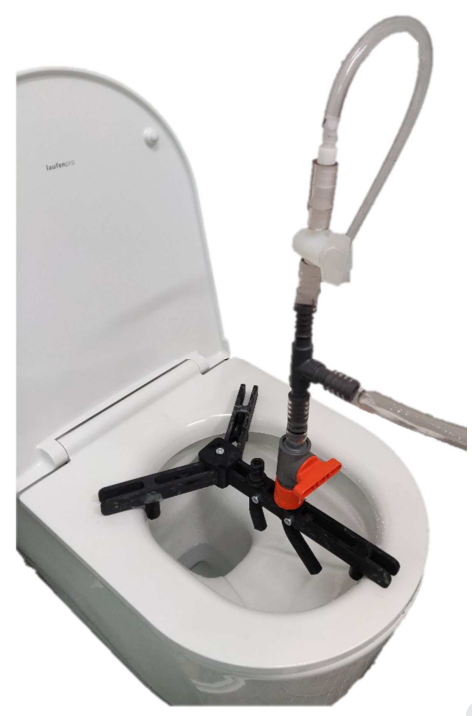

Figure 4: Setup used for real-tests: Prototype 3 in ceramic with Uromat and water supply.

\section{Results}

\subsection{Urine separation efficiency}

We first focus on the urine separation efficiency of the four models obtained at specific flow rates that we have chosen according to reference values in the literature, i.e. $33 \mathrm{ml} / \mathrm{s}$ and $26 \mathrm{ml} / \mathrm{s}$ for males and females, respectively [26,27]. A summary of the results for male and female and both inlet velocities of the four cases is given in Fig. 5 and 6.

Figure 5 shows a 3D view (center) and a side view (right) of the simulated male urine flow (33ml/s) obtained on Prototypes 1-4. It can be observed that the optimisation of tailored curvature radius and V-profile grooves lead to a substantial improvement in urine collection. In Prototype 4 the urine is channeled towards the curvature and then redirected around the curvature and into the urine collector, while other prototypes show significant detachment at the curvature. These detachments result both from small curvature radii (Fig. 5a,c) and high film thickness, provoked by a strong channeling of the fluid through the V-profile grooves (Fig. 5b). Both factors decrease the critical Weber number (Equation 1) and consequently lead to detachment of the fluid. 

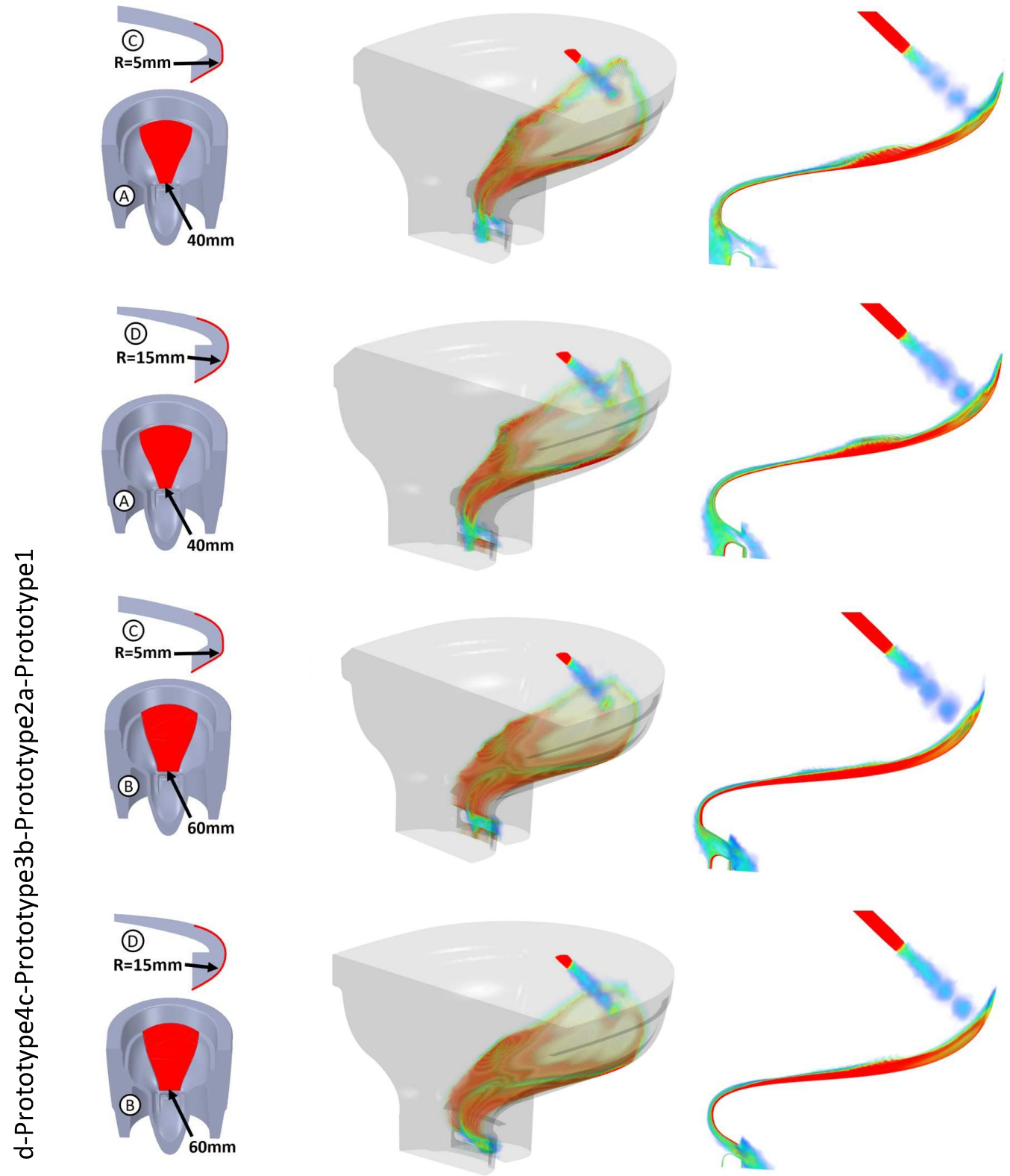

Figure 5: Geometries specifications (left), 3D view of liquid volume fraction (centre) and side views (right) of male urine flow $(33 \mathrm{ml} / \mathrm{s})$ in all four prototypes (a-d). All illustrations are at $4.6 \mathrm{~s}$ into the simulation.

To quantitatively compare the performance of each prototype, we show results of urine separation for the different cases in Fig. 6. The columns illustrate the urine separation for different simulation runs and prototypes, where the blue part is the amount of urine that was collected in the urine collector, while the orange part flowed 
into the flush water collector. A very clear improvement in urine separation can be observed from Prototype 1 to Prototype 4. This enhancement of performance was achieved by fine-tuning the curvatures radius and the Vprofile grooves which were implemented into the geometries.

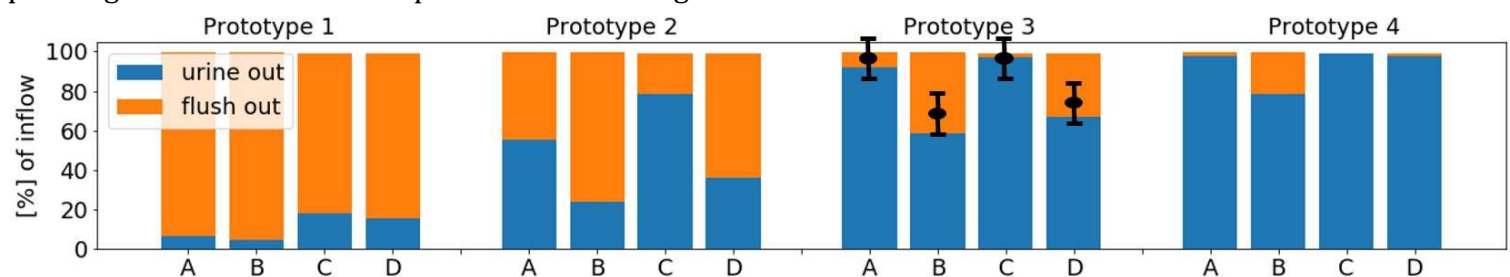

Figure 6: Urine separation efficiency of prototypes 1-4 for the different simulation runs. (A) female $25 \mathrm{ml} / \mathrm{s}$, (B) female $31 \mathrm{ml} / \mathrm{s}$, (C) male $27 \mathrm{ml} / \mathrm{s}$, (D) male $33 \mathrm{ml} / \mathrm{s}$. Data points of real-test on Prototype 3 are shown by the black symbols with corresponding error bars.

\subsection{Flush water}

The flush process was tested on all 4 prototypes to quantify the amount of flush water that flows into the urine collector and would mix with the urine collected there. Each prototype was tested upon a $3.2 \mathrm{~L}$ and a $4 \mathrm{~L}$ flush with a common flow intensity of $1 \mathrm{~L} / \mathrm{s}$. The results are summarized in Fig. 7. Each column represents one flush simulation, i.e. 3.2L and $4 \mathrm{~L}$, for all four prototypes. The figure shows the percentage of flush water that has entered the urine collector. Fig. 7 shows that an increased curvature radius has slightly negative effect on the flush-water separation, while the change of V-profile groove shows a negligible impact. Overall less then $2.5 \%$ (i.e. $<100 \mathrm{ml}$ for the $4 \mathrm{~L}$ flush) of flush water were drawn into the urine collector with little variation between prototypes which is satisfactory. The slightly lower percentage of separation for lower flow volumes results, as flush water is more likely to be drawn into the urine collector at the end of the flush process when flow rates are low.

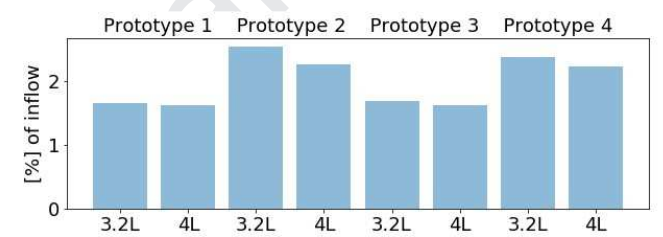

Figure 7: Percentage of flush water that was allocated in the urine collector for the four geometries.

\subsection{Validation experiments}

To validate our CFD-simulations, we conducted experiments on a physical model of Prototype 3. The comparison of liquid allocated in the urine collector from real test and simulations is shown in Fig. 6. It shows that the simulation results are within the range of real-tests. It can be noticed that the simulations tend to somewhat underestimate the separation efficiency. This might be a non-optimal turbulence model or slight differences in the experimental setup such as flow rate, positioning of the Uromat or impurities on the surface (pre-wetted, grease, dust). 


\section{Conclusion}

This study has successfully demonstrated that an efficient NoMix toilet can be designed by making use of the teapot effect. By tailoring the curvatures radius and V-profile grooves into the geometry, even at the worst case scenario (maximal urine flow rate implemented as a pulse wave), a very efficient urine separation could be achieved. With less then $2.5 \%$ of flush water entering the urine collector, this technology seems very promising and could possibly find broad application. The efficient source separation of urine allows the application of a broad range of recycling technologies and builds a further step towards sustainable sanitation systems.

\section{ACKNOWLEDGMENT}

This work was developed under the grant OPP1149059 funded by the Bill and Melinda Gates Foundation. The authors declare no conflict of interest

\section{References}

[1] J. R. Mihelcic, L. M. Fry, and R. Shaw, "Global potential of phosphorus recovery from human urine and feces," Chemosphere, vol. 84, no. 6, pp. 832-839, 2011.

[2] E. Andersson, "Turning waste into value: using human urine to enrich soils for sustainable food production in uganda," Journal of Cleaner Production, vol. 96, pp. 290-298, 2015.

[3] T. A. Larsen and W. Gujer, "Separate management of anthropogenic nutrient solutions (human urine)," Water Science and Technology, vol. 34, no. 3-4, pp. 87-94, 1996.

[4] J. A. Wilsenach and M. C. van Loosdrecht, "Integration of processes to treat wastewater and sourceseparated urine," Journal of Environmental Engineering, vol. 132, no. 3, pp. 331-341, 2006.

[5] B. Vinner as and H. Jo"nsson, "The performance and potential of faecal separation and urine diversion to recycle plant nutrients in household wastewater," Bioresource technology, vol. 84, no. 3, pp. 275-282, 2002.

[6] M. Maurer, W. Pronk, and T. Larsen, “Treatment processes for source-separated urine," Water research, vol. 40, no. 17, pp. 3151-3166, 2006.

[7] J. C. Berndtsson, "Experiences from the implementation of a urine separation system: Goals, planning, reality," Building and environment, vol. 41, no. 4, pp. 427-437, 2006.

[8] J. Lienert, T. Bürki, and B. I. Escher, "Reducing micropollutants with source control: substance flow analysis of 212 pharmaceuticals in faeces and urine," Water Science and Technology, vol. 56, no. 5, pp. 87-96, 2007.

[9] M. E. Borsuk, M. Maurer, J. Lienert, and T. A. Larsen, "Charting a path for innovative toilet technology using multicriteria decision analysis," 2008.

[10] J. Lienert and T. A. Larsen, "Considering user attitude in early development of environmentally friendly technology: A case study of nomix toilets," 2006.

[11] J. Lienert and T. A. Larsen, "High acceptance of urine source separation in seven european countries: a review," Environmental science \& technology, vol. 44, no. 2, pp. 556-566, 2009.

[12] J. Lienert, M. Haller, A. Berner, M. Stauffacher, and T. A. Larsen, "How farmers in switzerland perceive fertilizers from recycled anthropogenic nutrients (urine)," Water Science and Technology, vol. 48, no. 1, pp. 47-56, 2003.

[13] www.vuna.ch/aurin. 
[14] L. Rossi, J. Lienert, and T. Larsen, "Real-life efficiency of urine source separation," Journal of environmental management, vol. 90, no. 5, pp. 1909-1917, 2009.

[15] H. Sozer, "Improving energy efficiency through the design of the building envelope," Building and environment, vol. 45, no. 12, pp. 2581-2593, 2010.

[16] Z. Gou and X. Xie, "Evolving green building: triple bottom line or regenerative design?," Journal of Cleaner Production, vol. 153, pp. 600-607, 2017.

[17] J. Hu, Y. Sun, and M. Liu, "Numerical simulation of two-phase flow in wash-down toilet flush process," Journal of System Simulation, vol. 20, no. 6, pp. 1599-1602, 2008.

[18] J. G. Hu, Y. S. Sun, and Z. R. Zhang, "Numerical simulation and experimental validation of threedimensional unsteady multi-phase flow in flushing process of toilets," in Applied Mechanics and Materials, vol. 444, pp. 304-311, Trans Tech Publ, 2014.

[19] S. Zhao, Z. Liu, and Z. Peng, "Optimized design of toilet siphon pipeline based on fluent and its validated test,” Journal of System Simulation, vol. 20, no. 16, pp. 4412-4416, 2008.

[20] C. Duez, L. Bocquet, C. Ybert, and C. Clanet, "Beating the teapot effect," Phys. Rev. Lett. vol, vol. 4, no. arXiv: 0910.3306, p. 084503, 2009.

[21] W. Bouwhuis and J. H. Snoeijer, "The effect of surface wettability on inertial pouring flows," arXiv preprint arXiv:1507.05931, 2015.

[22] ANSYS, Fluent, “Ansys fluent, products release 19.1.”

[23] EOOS, “Eoos urinator,” 2019. http://www.urinetrap.com/src/assets/img/ Urinator.pdf.

[24] Solidworks, "Solidworks, education edition 2016."

[25] ANSYS, SpaceClaim, “Ansys spaceclaim, products release 19.1.”

[26] B. Haylen, D. Ashby, J. R. Sutherst, M. Frazer, and C. West, "Maximum and average urine flow rates in normal male and female populationsthe liverposl nomograms," British journal of urology, vol. 64, no. 1, pp. 30-38, 1989.

[27] V. Kumar, J. V. Dhabalia, G. G. Nelivigi, M. S. Punia, and M. Suryavanshi, "Age, gender, and voided volume dependency of peak urinary flow rate and uroflowmetry nomogram in the indian population," Indian journal of urology: IJU: journal of the Urological Society of India, vol. 25, no. 4, p. 461, 2009. 
Highlights for review:

- Separation of urine from feces in NoMix toilets bears a large potential for mitigating problems associated with the efficient reuse of excreta.

- Acceptance and implementation of NoMix toilets has been limited due to the design of such toilets.

- We present a new design idea that is more likely to be accepted by the consumer since it doesn't require any change of behavior from the user nor does it include any moving parts.

- We test this design via CFD-simulations and validate the model conducting experiments on a physical structure.

- We achieve very high urine separation with minimal flush water mixing due to tailored design. 


\section{Declaration of interests}

$\bigotimes$ The authors declare that they have no known competing financial interests or personal relationships that could have appeared to influence the work reported in this paper.

$\square$ The authors declare the following financial interests/personal relationships which may be considered as potential competing interests: 
Joel Gundlach:

Conceptualization, Methodology, Software, Validation, Formal analysis, Investigation, Writing - Original Draft, Writing - Review \& Editing, Visualization

Michael Bryla:

Conceptualization, Methodology, Validation, Investigation, Visualization

Tove A. Larsen:

Methodology, Writing - Review \& Editing, Visualization

Lotte Kristoferitsch:

Conceptualization, Resources, Writing - Review \& Editing, Project administration

Harald Gündl:

Conceptualization, Methodology, Resources, Writing - Review \& Editing, Visualization, Project administration, Funding acquisition

Markus Holzner:

Conceptualization, Methodology, Formal analysis, Resources, Writing - Review \& Editing, Visualization, Supervision, Project administration 